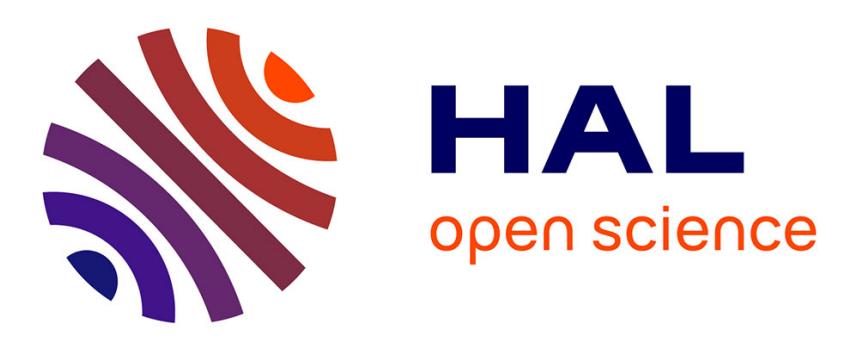

\title{
Metabolic Positron Emission Tomography Response to Gamma Knife of the Ventral Intermediate Nucleus in Essential Tremor
}

\author{
Antoine Verger, Tatiana Witjas, Romain Carron, Alexandre Eusebio, \\ Emmanuelle Boutin, Jean-Philippe Azulay, Jean Régis, Eric Guedj
}

\section{To cite this version:}

Antoine Verger, Tatiana Witjas, Romain Carron, Alexandre Eusebio, Emmanuelle Boutin, et al.. Metabolic Positron Emission Tomography Response to Gamma Knife of the Ventral Intermediate Nucleus in Essential Tremor. Neurosurgery, 2019, 84 (6), pp.E294-E303. 10.1093/neuros/nyy340 . hal-02479731

\section{HAL Id: hal-02479731 \\ https://hal.science/hal-02479731}

Submitted on 21 Apr 2020

HAL is a multi-disciplinary open access archive for the deposit and dissemination of scientific research documents, whether they are published or not. The documents may come from teaching and research institutions in France or abroad, or from public or private research centers.
L'archive ouverte pluridisciplinaire HAL, est destinée au dépôt et à la diffusion de documents scientifiques de niveau recherche, publiés ou non, émanant des établissements d'enseignement et de recherche français ou étrangers, des laboratoires publics ou privés. 


\section{METABOLIC PET RESPONSE TO GAMMA KNIFE OF THE VENTRAL INTERMEDIATE NUCLEUS IN ESSENTIAL TREMOR}

Antoine Verger MD, $\mathrm{PhD}^{1,2,3}$; Tatiana Witjas $\mathrm{MD}^{4,5}$; Romain Carron $\mathrm{MD}, \mathrm{PhD}^{6,7}$;

Alexandre Eusebio MD, $\mathrm{PhD}^{4,5}$; Emmanuelle Boutin $\mathrm{MD}^{4,5}$; Jean-Philippe Azulay MD, $\mathrm{PhD}^{4,5}$; Jean Regis $\mathrm{MD}^{6,7}$; Eric Guedj $\mathrm{MD}, \mathrm{PhD}^{1,5,8}$.

${ }^{1}$ Department of Nuclear Medicine, Assistance Publique-Hôpitaux de Marseille, Aix-Marseille Université, Timone University Hospital, France;

${ }^{2}$ Department of Nuclear Medicine \& Nancyclotep Imaging platform, CHRU Nancy, Lorraine University, France;

${ }^{3}$ IADI, INSERM, UMR 947, Lorraine University, Nancy, France;

${ }^{4}$ Department of Neurology and Movement Disorders, Assistance Publique-Hôpitaux de Marseille, Aix-Marseille Université, Timone University Hospital, France;

${ }^{5}$ Aix-Marseille Univ, CNRS, Centrale Marseille, Institut Fresnel, Marseille, France;

${ }^{6}$ Department of Functional and Stereotactic Neurosurgery and Radiosurgery, Assistance Publique-Hôpitaux de Marseille, Aix-Marseille Université, Timone University Hospital, France;

${ }^{7}$ INSERM, UMR 1106, Aix-Marseille Université, Marseille, France;

${ }^{8}$ CERIMED, Aix-Marseille Université, Marseille, France.

\section{Address for correspondence:}

Eric Guedj, M.D., Ph.D.

eric.guedj@ap-hm.fr

Service Central de Biophysique et Médecine Nucléaire, Hôpital de la Timone, 264 rue Saint Pierre, 13005 Marseille, France.

Tel.: +33-491385558; Fax: +33-491384769

\section{Conflict of Interest: None}

Funding: This work has been carried out in the framework of DHU-Imaging thanks to the support of the A*MIDEX project ( $\mathrm{n}^{\circ}$ ANR-11-IDEX-0001-02), funded by the « Investissements d'Avenir » French Government programme and managed by the French National Research Agency (ANR). This work was also supported by Inserm (Centre d'Investigation Clinique, CIC, Hôpital de la Conception, Marseille) and AP-HM (PHRC 2007/09). 


\section{Abstract}

Background: To understand the substrates of response and non-response and to identify potential biomarkers for the selection and follow-up of patients with essential tremor (ET) treated with gamma knife of the ventral intermediate nucleus (GKVIM).

Objective: This observational study aimed to characterize PET changes in the metabolism of glucose and metabolic connectivity in patients with ET treated by GKVIM.

Methods: Forty-two patients with right ET were referred to ${ }^{18}$ F-FDG PET imaging before and after left GKVIM. Statistical Parametric Mapping T-score map comparisons were performed between pre- and post-GKVIM groups and between clinical responders and non-responders. Metabolic connectivity was evaluated by the inter-regional correlation analysis method.

Results: After GKVIM, patients with ET exhibited decreased left thalamic metabolism, which was associated with remote metabolic decreases in the right cerebellum, left temporal gyri, and bilateral frontal gyri ( $\mathrm{p}<0.05$, family-wise error-corrected). Additionally, non-responders $(n=7)$ showed metabolic decreases in the right temporo-occipital area $(\mathrm{p}<0.005$ corrected for cluster volume) after GKVIM. The metabolism in this area was already reduced in nonresponders before treatment in comparison to that in responders and was predictive of future response (sensitivity: $89 \%$; specificity: $71 \%$ ). In non-responder patients, strong connectivity between the left thalamus and right temporo-occipital area was found before GKVIM and was lost after treatment, whereas this connectivity remained weak and stable in responders.

Conclusions: These findings could lead to better knowledge of the variability in the metabolic PET profiles among patients with ET, particularly the integration of ${ }^{18}$ F-FDG PET imaging in the pretherapeutic evaluation of patients with refractory ET candidates for GKVIM.

Running title: PET in radiosurgery of essential tremor

Key-words: Connectivity; Essential Tremor; FDG PET; Gamma knife of the ventral intermediate nucleus; Predictive value 


\section{Introduction}

Essential tremor (ET) increases in both incidence and severity with ageing ${ }^{1}$, leading to severe tremors that are not well controlled with standard treatments ${ }^{2}$. In this context, deep brain stimulation (DBS) of the ventral intermediate nucleus (Vim) and radiofrequency thalamotomy have been established as neurosurgical options for medically refractory tremors ${ }^{3}$. However, these procedures are technically difficult and carry great risk in elderly patients ${ }^{4}$. Gamma knife of the Vim (GKVIM) is a radiosurgical treatment with a low rate of complications ${ }^{5}$, and a prospective study demonstrated that unilateral GKVIM is a safe and efficient treatment for severe medically refractory ET ${ }^{6}$. However, the exact relationship between locally induced lesions and the clinical improvement remains unclear, particularly because of remote brain effects ${ }^{7-9}$. For instance, in patients with ET who were treated with MR imaging-guided focused ultrasound ${ }^{10}$, diffusion-tensor imaging (DTI) revealed changes over time not only at the site of the thalamotomy but also in distributed areas of the brain, and only remote DTI changes were correlated with the clinical improvement. On the other hand, ET is a widely heterogeneous condition. There is a need for standardization of ET pathology across studies ${ }^{11}$. Along this line, research of biomarkers is of great interest to better understand the pathophysiological mechanisms involved in ET.

Unfortunately, a proportion of non-improved patients have been reported after GKVIM, corresponding to $24 \%$ of treated patients ${ }^{6}$. Moreover, one case of a non-improved patient was found despite MR evidence of necrosis-induced lesions ${ }^{6}$, highlighting the complexity between local changes and clinical improvement. At this time, there is no predictive biomarker that distinguishes between future clinical responders and non-responders before GKVIM. Neuroimaging, including multimodal MRI and PET, could provide additional insights into ET physiopathology. In this vein, recent MRI studies have reported the involvement of the visuo-motor network in ET in VBM studies ${ }^{12,13}$ and fMRI studies ${ }^{14,15}$.

The use of cerebral ${ }^{18} \mathrm{~F}$-fluorodesoxyglucose positron emission tomography $\left({ }^{18} \mathrm{~F}\right.$-FDG PET $)$ has been proposed to determine treatment responses in various neurological disorders ${ }^{16,17}$. ${ }^{18}$ F-FDG PET is used to precisely evaluate resting-state synaptic activity by measuring the cerebral metabolic rate of glucose (CMRGlc) ${ }^{18}$ and has been widely used in the context of movement disorders ${ }^{19}$. Moreover, ${ }^{18}$ F-FDG PET determines the metabolic connectivity of cerebral networks using inter-regional correlation analysis (IRCA) ${ }^{20,21}$. However, the 
technical performance of PET instrumentation is less favourable, despite an original targeting of the glucose consumption that is known to precede the transient relative decrease in the BOLD signal without any magnetic limitations ${ }^{18}$ and rather better signal-to-noise ratios, variance concentrations and out-of-sample replication at the individual level than fMRI ${ }^{22}$. This study aims to characterize changes in the CMRGlc and metabolic connectivity in patients with ET treated with GKVIM. The objective is to determine the metabolic networks involved, to understand the metabolic substrates of response and non-response, and to identify potential biomarkers for selection and follow-up.

We hypothesize that a particular network involving the thalamus could lead to distinct responses to GKVIM, with potentially different metabolic profiles between responders and non-responders. 


\section{Materials and Methods}

\section{Subjects}

In this observational study, patients with ET were recommended for ${ }^{18}$ F-FDG PET imaging before and after left GKVIM treatment. Between April 2012 and March 2015, 221 consecutive patients who were referred to

were prospectively evaluated for GKVIM as a treatment for medically refractory ET. ET was defined as a condition in which the upper limbs and, less commonly, the head, face, voice, trunk, and lower limbs exhibit mixed postural and kinetic tremors without other neurologic abnormalities ${ }^{23}$. Patients with typical Parkinson's disease were excluded. Patients were treated with GKVIM in cases where it was impossible to perform Vim DBS for any medical or surgical reason or in rare cases of patient refusal. Only patients referred for left GKVIM were considered for this study. Response was defined according to the Fahn-Tolosa-Marin Tremor Rating Scale ${ }^{24}$, with a cut-off of $45 \%$ for improvement ${ }^{6}$. The MRI follow-up consisted of serial scans performed 6 and 12 months after radiosurgery to assess the procedure and to identify subsequent induced lesions. For each patient, the one-year follow-up MRI scan was coregistered with stereotactic images using the Leksell GammaPlan (LGP) software (Elekta Instruments, Stockholm). A lesion was defined as a thalamic area showing T2-weighted and fluid attenuated inversion recovery (FLAIR) hyperintense-limited signal changes and a postcontrast ring-shaped lesion at 12 months, with the classical "cockade" pattern. On the other hand, a patient with no thalamic lesions was defined as having no contrast enhancement and no FLAIR changes in the thalamic area at 12 months. The stereotactic coordinates of the centre of the lesion, defined as the centre of the hyposignal area, were determined with LGP and compared with the set of coordinates of the target.

${ }^{18}$ F-FDG PET was performed $60( \pm 84)$ days before GKVIM and $484( \pm 85)$ days after GKVIM. We excluded patients under 18 years of age, patients with psychiatric or other neurologic conditions, and non-right-handed patients. Cognitive performance was assessed using the Mattis Dementia Rating Scale (MDRS) ${ }^{25}$. All patients participated with written informed consent. 
The study was approved by the local ethics committee and was performed in compliance with both national legislation and the Declaration of Helsinki. The final population involved fortytwo right-handed patients, as described in Table 1.

Data from thirty-one healthy subjects with similar demographic characteristics to those of the patients were extracted from a local normal ${ }^{18}$ F-FDG PET database approved by the local ethics committee ( $71.4 \pm 6.5$ years, 16 women; $p=0.12$ for age, and 0.85 for gender) (Clinical

Trials Ref.: (). The healthy subjects were free from neurological/psychiatric diseases and cognitive complaints and had normal morphometric brain MRI (T1-weighted, T2-weighted and FLAIR sequences) scans.

\section{GKVIM protocol}

The radiosurgical procedures were all performed by the same neurosurgeon ( $\square$. Patients were unilaterally treated in the left thalamus. As described previously ${ }^{6}$, the Leksell@ coordinate frame (Elekta AB, Stockholm, Sweden) was applied under local anaesthesia. Special care was taken to place the stereotactic frame parallel to the orientation of the anterior commissure-posterior commissure (AC-PC) line to minimize head tilt and rotation. Treatment planning was performed using the Leksell GammaPlan software (versions 8.3.1 up to 10.1.1). The centre of the target, determined by Guiot's diagram, was placed $2.5 \mathrm{~mm}$ above the ACPC line, $11 \mathrm{~mm}$ lateral to the wall of the third ventricle, and three-twelfths of AC-PC distance on the Guiot scheme in front of the PC. It should be noted that variations in the y plane of the "Guiot" target were related to individual variations in the CA-CP length, having a mean of $25.11 \mathrm{~mm}(23-28.30 \mathrm{~mm})$. In addition, a unique 4-mm isocentre was used for beam blocking to optimize the gradient of the dose towards the internal capsule. The prescribed dose was 130 Gy (maximum dose) for all patients.

\section{${ }^{18}$ F-FDG PET Acquisition and Analysis}

${ }^{18}$ F-FDG PET scans were performed before and after GKVIM for each patient, under the same conditions as those for the healthy subjects. An integrated PET/CT camera (Discovery ST, GE Healthcare, Waukesha, WI) was used with an axial resolution of $6.2 \mathrm{~mm}$, allowing 47 3.27-mm thick contiguous transverse sections of the brain. ${ }^{18} \mathrm{~F}-\mathrm{FDG}$ (150 MBq) was injected intravenously during both awake and resting states with each patient's eyes closed in a quiet 
environment. Image acquisition started $30 \mathrm{~min}$ after injection and ended 15 min thereafter. Images were reconstructed using the ordered subsets expectation maximization algorithm with 5 iterations and 32 subsets and were corrected for attenuation using a CT transmission scan.

Whole-brain statistical analysis was performed at the voxel-level using the SPM8 software (Wellcome Department of Cognitive Neurology, University College, London, UK) to compare the entire group of patients before and after surgery first then to compare responders and non-responders. The PET images were spatially normalized onto the Montreal Neurological Institute $(\mathrm{MNI})$ atlas. The dimensions of the resulting voxels were $2 \times 2 \times 2 \mathrm{~mm}$. The images were then smoothed with a Gaussian filter ( $8 \mathrm{~mm}$ full-width at half-maximum) to blur individual variations in gyral anatomy and to increase the signal-to-noise ratio. The between-groups SPM(T) maps were obtained at a height threshold (voxel-level significance) of $\mathrm{p}<0.05$, corrected for multiple comparisons at the cluster level (FWE: family-wise error method), with a minimum of 25 voxels per cluster. Secondarily, if no change was initially found using the FWE correction a less conservative voxel threshold of $\mathrm{p}<0.005$ was used, only corrected for the cluster volume to avoid type II errors as recommended ${ }^{26}$. Age and gender were used as nuisance variables. Comparison analyses were performed using a flexible factorial model (group X time) between the following: (i) pre- vs. post-GKVIM groups to determine the metabolic changes involved after GKVIM (Time Factor); (ii) non-responder patients vs. responder patients, before and after GKVIM (Group Factor); and (iii) between these two time-points (Group X Time Factors) to identify the metabolic substrates of response and non-response, potential biomarkers to select and follow-up patients. Proportional scaling was applied, giving the same global value to each PET examination to correct for individual variations in global brain metabolism. The anatomical localization of the most significant voxels was then identified using the MNI (Montreal Neurological Institute) atlas. The mean values of the CMRGlc were extracted at the individual level for each cluster found.

To evaluate the metabolic connectivity of the metabolic cluster(s) previously identified in the intergroup comparison, IRCA was performed according to the validated procedure ${ }^{20}$. Briefly, the mean values of the CMRGlc were used as interacting covariates for the comparison between connectivity in the responders and that in the non-responders. Correlation coefficients were thereafter calculated between the mean values of the CMRGlc of the significant cerebral areas from the previous between-group analysis. 


\section{Statistical Analysis}

Quantitative variables are expressed as the means \pm standard deviations and categorical variables as percentages. The statistical parameters for the SPM analyses are detailed above. Student's t-tests were performed for mean comparisons in the normally distributed quantitative variables, while Chi-square tests were performed for the categorical variables. Spearman coefficients were used to estimate the correlations between the mean values of the CMRGlc in the clusters identified in the intergroup comparison. Comparisons between correlation coefficients were performed with Fisher tests ${ }^{27}$ or Steiger tests when appropriate 28 . There were not any missing data in this study. P-values of 0.05 were considered significant.

\section{Results}

\section{Patient characteristics}

Forty-two right-handed patients (71.6 \pm 5.9 years; fourteen women) underwent unilateral left GKVIM. Thirty-five patients (83\%) were clinical responders (clinical tremor improvement $>45 \%$ according to the Fahn-Tolosa-Marin Tremor Rating Scale ${ }^{29}$, with 5 experiencing $100 \%$ improvement) with a mean clinical tremor improvement of $64.2 \%$ in the whole group. In the responders subgroup, the mean clinical improvement was $76.3 \%$. No difference was found in the clinical characteristics between the responders and the non-responders (age, gender, diabetes mellitus status, MDRS total score and tremor severity before GKVIM; $p>0.05$ ) or in terms of the coordinates of the stereotactic target, of the lesion, and the difference between the target and the lesion in each direction ( $p>0.05$ ). In addition, no difference was observed in the delay of the pre-surgical FDG PET and GKVIM and the post-surgical FDG PET and GKVIM between responders and non-responders $(\mathrm{p}=0.35$; Table 1$)$.

Thirty-two patients (76\%) presented a left thalamic lesion during the MRI follow-up, with a sensitivity of $86 \%$, a specificity of $71 \%$ and an accuracy of $83 \%$ for the clinical response. The average diameter of the thalamic lesions induced by GKVIM was $6.6( \pm 7.6) \mathrm{mm}$ with a range of 2-12.8 mm. Two patients (5\%) showed hyper-responses on the MRI (defined as an MRI lesion $\geq 12 \mathrm{~mm}$ ). These two patients were clinical responders. The occurrence of a thalamic 
MRI lesion was different between clinical responders and non-responders, as the lesion was statistically associated with the clinical response $(\mathrm{p}<0.01)$. Nevertheless, two patients with severe tremor were clinical non-responders and had thalamic lesions visible on MRI, while 5 patients were clinical responders and had no responses visible on MRI. It should be noted that no difference in the coordinates was observed between responders and non-responders for the "Guiot" target ( $>0.45)$, for the MRI lesion ( $>>0.62)$, or between the "Guiot" target and the MRI lesion ( $\mathrm{p}>0.05)$.

\section{Whole-group analysis of the patients}

In comparison to the baseline metabolism found before treatment, after GKVIM the patients showed significantly decreased metabolism within the left thalamus $(\mathrm{p}<0.05$ corrected for multiple comparisons using FWE; Figure 1; Table 2). Significant metabolic decreases were also identified remotely in the right cerebellum, the left superior and middle temporal gyri (BA21-22-38), and the bilateral middle and inferior frontal gyri (BA10-46). All these clusters were already hypometabolic before the treatment in comparison to those of the healthy control group $(\mathrm{p}<0.01)$.

\section{Analysis in responder vs. non-responder patient's subgroups}

Before GKVIM, the basal metabolism was different between the two subgroups of patients, with hypometabolism being observed in the right retrosplenium and the posterior cingulate cortex (BA29-30-31) in non-responders in comparison to that in responders, as shown in Figure 2 ( $\mathrm{p}<0.005$, corrected for cluster volume). After GKVIM, this hypometabolism spread to other areas in the non-responders, including the right lingual gyrus (BA18-19) $(p<0.005$, corrected for cluster volume; Figure 2; Table 2). Hypometabolism was confirmed after comparison with the healthy controls ( $\mathrm{p}<0.005$, corrected for cluster volume) for both subgroups of patients.

In comparison to the pretherapeutic evaluation and to the responders, decreased metabolism in the right temporo-occipital area was found in non-responders after GKVIM, including the right lingual, inferior occipital and fusiform gyri (BA17-18-19-37) $(\mathrm{p}<0.005$, corrected for cluster volume; Time X Group Factors; Figure 2; Table 2). Hypometabolism was confirmed 
in comparison to the healthy control group ( $\mathrm{p}<0.005$, corrected for cluster volume) in both responders and non-responders.

On the one hand, and despite a trend, the local left thalamic PET metabolism obtained after treatment was not significantly associated with clinical response in either responders or nonresponders $(\mathrm{p}=0.09)$. On the other hand, the remote PET hypometabolism found in temporooccipital areas before treatment in non-responders was significantly associated with clinical response ( $\mathrm{p}<0.01$, for comparison between responders and non-responders), constituting a predictive metabolic biomarker in the preoperative state.

The mean CMRGlc value of the right retrosplenium and posterior cingulate cluster showed significant predictive value before treatment regarding the clinical response to GKVIM. The receiver operating characteristic curve is reported in Figure 3. A mean value of 65 was, for example, associated with an $89 \%$ sensitivity, $71 \%$ specificity and $86 \%$ accuracy for response prediction. The responders identified by PET were in accordance with the clinical data in 6 cases and with the MRI data in the 5 other cases. Furthermore, imaging detection of a lesion by MRI was not associated with local PET changes ( $\mathrm{p}=0.57)$, and no correlation between the volume of lesion detected on MRI and local PET changes was observed ( $p=0.17)$, suggesting that complementary information was provided by both modalities. Thus, the accuracy of the imaging detection of a lesion by MRI was $83.3 \%$. This accuracy was not improved by the addition of the lesion volume observed via MRI (accuracy of 83.3\%). The accuracy of the PET imaging could be improved by the addition of thalamic metabolism before treatment (accuracy of 90.5\%). Interestingly, the combination of imaging detection of a lesion by MRI and PET temporo-occipital metabolism before treatment reached an accuracy of $90.5 \%$, strengthening the hypothesis of complementarity between these two modalities. Illustrating this hypothesis, the combination of thalamic and temporo-occipital metabolism before and after treatment and the imaging detection of a lesion by MRI lead to an accuracy of $100 \%$.

Metabolic connectivity was thereafter studied between the thalamic target and these remote effects on temporo-occipital areas. The IRCA detected different correlations in the 3 groups of subjects concerning the connectivity of the left thalamus and right temporo-occipital areas. At baseline, the connectivity in the non-responders was highly strong (Spearman coefficient $r$ of $0.81, \mathrm{p}=0.18$ ) in comparison to that in the responders $(\mathrm{r}=0.05, \mathrm{p}=0.93)$, and was found to be at a lower level in the healthy subjects $(r=0.54, p=0.02)(p=0.03$, for the comparison of the correlation coefficients between responders and non-responders). After GKVIM, the connectivity in the responders was statistically unchanged $(r=0.26, p=0.05)$, while the 
connectivity was lost in non-responders $(r=0.05, p=0.94 ; p>0.24$ for the comparison of correlation coefficients between the two subgroups of patients, as well as within each group). A summarized diagram of the PET results is available in Figure 4. 


\section{Discussion}

After left GKVIM treatment, the entire group of patients with ET unsurprisingly exhibited decreased left thalamic metabolism in comparison to the metabolism found before treatment. Moreover, this decreased metabolism was associated with remote metabolic decreases in the right cerebellum, the left superior and middle temporal gyri, and the bilateral middle and inferior frontal gyri. In addition to its ability to better understand the mechanisms involved in essential tremor physiopathology and its physiological response, PET imaging provides a diagnostic performance that is predictive and complementary to MRI data.

Otherwise, as previously reported in at least one case ${ }^{6}$, we found some discrepancies between clinical and radiological responses, which were distributed as follows: 5 clinical responses without thalamic MRI lesions and 2 clinical non-responses with MRI lesions. The first discrepancy showed that the clinical effect was not systematically associated with a morphological lesion. This observation reinforced the hypothesis that the mechanisms of action of GKVIM act as a neuromodulation therapy ${ }^{30}$, thus having possible metabolic remote effects. In line with this, ${ }^{18}$ F-FDG PET appears to be an effective functional imaging procedure that is complementary to morphological MRI. The second discrepancy, though less frequent, may be explained by the anatomo-metabolic variability among the subjects, underlying distinct clinical responses for similar morphological lesions. The comparison of PET data between responders and non-responders provides an opportunity to better understand these discrepancies and to consolidate these hypotheses.

Our study found that $83 \%$ of the patients were clinical responders after GKVIM, a finding that was in line with previous studies ${ }^{5,31}$. Interestingly, no difference in the left thalamic metabolic changes was found after GKVIM between responders and non-responders. This important result goes against the single local effect hypothesis explaining the clinical responses ${ }^{10}$. On the other hand, decreased metabolism in the right temporo-occipital area was found in non-responders after GKVIM in comparison to that in the pretherapeutic evaluation and to that in responders. This metabolic cluster was already reduced before treatment in comparison to that in the responders and to that in the healthy subjects. Reduced activity in the temporo-occipital area has already been described in patients with ET in comparison with that in healthy controls in functional imaging studies, including those utilizing resting-state fMRI ${ }^{32}$ and ${ }^{18}$ F-FDG PET ${ }^{33}$. Similarly, in our study, non-responders showed a relatively focal decrease in metabolism in the right retrosplenium and posterior cingulate area before GKVIM and in comparison to responders. Importantly, the CMRGlc of this cluster was 
predictive of the clinical response to GKVIM. The retrosplenium and posterior cingulate areas are known to be associated with the visual cerebral network and to play a key role in visuospatial orientation ${ }^{34}$.

Interestingly, a recent fMRI study showed a widespread visually sensitive functional network related to symptoms of essential tremor, a finding that aligns with our results ${ }^{35}$

On the other hand, metabolic connectivity in patients with ET has been explored, particularly in terms of responders and non-responders, through the well-known connectivity between the thalamus and temporo-occipital areas ${ }^{36}$. Connectivity between the thalamus and these areas was also found here but differed between the subgroups of ET patients, strengthening the hypothesis of an existing metabolic variability among these subjects. Indeed, non-responders presented strong connectivity between these two areas before GKVIM, possibly reflecting a compensatory mechanism. This connectivity markedly decreased after GKVIM, possibly corresponding to a loss of a metabolic compensation, and may, at least partly, explain the non-response to treatment. In line with this, a voxel-based morphometry study ${ }^{37}$ revealed a relative expansion of grey matter bilaterally in the temporo-parietal junction and right middle occipital cortex in patients with ET, which might represent adaptive reorganization, and compensate skilled movements in cases of trembling. These findings highlight the specific link between the motor and visual cerebral networks. The increased connectivity observed between the thalamus and temporo-occipital areas aligns with this statement. The meaning of the temporo-occipital hypometabolism is, nevertheless, more confusing in the resting state. The first hypothesis would state it is the result of a compensatory mechanism, taking into account that a hypometabolic area at rest could be more easily activated in case of a compensatory-task. In this context, hypometabolism during the resting condition has been associated with hyperactivation in the same regions during an fMRI task ${ }^{38}$. This hypometabolism could alternatively correspond to a more severe disorder, including an additional visual impairment, with a less favourable response to treatment. Illustrating this second hypothesis, an increase in visual feedback worsened tremor in the 4-12 Hz range in patients with essential tremor and was associated with abnormal changes in BOLD signal amplitude and entropy in the cerebello-thalamo-motor cortical pathway and in visual and parietal areas in a recent fMRI study ${ }^{35}$. In line with this, a precise evaluation of visual function appears necessary to better characterize the clinical heterogeneity of patients with ET. Otherwise, the right predominance of abnormalities found in non-responders should 
certainly be related, on the one hand, to the right hemispheric predominance of visual areas ${ }^{39}$, and, on the other hand, to the right ET dominance of our patients.

In the present study, PET imaging provided pretherapeutic information, whereas the information provided by MRI was only post-therapeutic. In line with this, the analysis of remote temporo-occipital PET metabolism is associated with good diagnostic performance (accuracy of 86\%) in determining clinical outcome before treatment. Thus, this analysis should provide an additional target for further potential surgeries in essential tremor. Moreover, PET imaging underlines the essential function of the visuo-motor system in this pathology, which could be a potential target in the rehabilitation processes. This database could be further used to stress the long-term effectiveness of GKVIM by discussing the locations of shots and lesions and incorporating multimodal MRI. Further studies using fMRI could especially provide additional information, particularly in relation to the underlying involvement of the visuo-motor system in ET pathophysiology and the effective response within this system obtained after GKVIM.

\section{Limitations}

Our study had several limitations. First, there was no randomization or waiting list that allowed direct comparisons to a control population of non-treated ET patients. Moreover, the subgroups of patients were unequal, with a small number of non-responder patients. Even though the patients were not cognitively impaired according to our evaluations, all cognitive functions were not explored, particularly those involved in visuo-spatial functions. Only righthanded patients and left GKVIM treatments were included; therefore, the results cannot be extended to patients with other characteristics. Finally, the findings should be replicated in a separate and larger cohort.

\section{Conclusion}

Overall, this metabolic ${ }^{18}$ F-FDG PET imaging study revealed changes in the CMRGlc after left GKVIM for right ET that involved a left thalamic target and, remotely, a corticocerebellar network. Moreover, in non-responders, increased connectivity between the thalamus and the temporo-occipital area and a prior metabolic feature involving hypometabolism of the visuospatial cortex prevailed. This metabolic pattern of the nonresponders could condition the clinical response to the radiosurgical procedure, suggesting a specific link between the motor and visual cerebral networks, and may help to determine biomarkers of response and follow-up after GKVIM. These findings could u lead ultimately 
to a better knowledge of the variability in the metabolic PET profiles among patients with ET. In addition to the ability of PET to better understand ET pathophysiological mechanisms and phenotypical heterogeneity, PET imaging provides a predictive pretherapeutic performance that is complementary to the MRI follow-up of post-therapeutic responses. This promising multimodality approach should be experimented on further in larger studies. 


\section{References}

1. Benito-León J, Bermejo-Pareja F, Morales J-M, Vega S, Molina J-A. Prevalence of essential tremor in three elderly populations of central Spain. Mov Disord Off J Mov Disord Soc. 2003;18(4):389-394. doi:10.1002/mds.10376

2. Elias WJ, Shah BB. Tremor. JAMA. 2014;311(9):948-954. doi:10.1001/jama.2014.1397

3. Schuurman PR, Bosch DA, Bossuyt PM, et al. A comparison of continuous thalamic stimulation and thalamotomy for suppression of severe tremor. N Engl J Med. 2000;342(7):461-468. doi:10.1056/NEJM200002173420703

4. Koller WC, Pahwa PR, Lyons KE, Wilkinson SB. Deep brain stimulation of the Vim nucleus of the thalamus for the treatment of tremor. Neurology. 2000;55(12 Suppl 6):S29-33.

5. Ohye C, Higuchi Y, Shibazaki T, et al. Gamma knife thalamotomy for Parkinson disease and essential tremor: a prospective multicenter study. Neurosurgery. 2012;70(3):526-535; discussion 535-536. doi:10.1227/NEU.0b013e3182350893

6. Witjas $\mathrm{T}$, Carron R, Krack $\mathrm{P}$, et al. A prospective single-blind study of Gamma Knife thalamotomy for tremor. Neurology. 2015;85(18):1562-1568. doi:10.1212/WNL.0000000000002087

7. Hayashi $\mathrm{T}$, Fukuyama $\mathrm{H}$, Katsumi $\mathrm{Y}$, et al. Cerebral glucose metabolism in unilateral entorhinal cortex-lesioned rats: an animal PET study. Neuroreport. 1999;10(10):2113-2118.

8. Meguro K, Blaizot X, Kondoh Y, Le Mestric C, Baron JC, Chavoix C. Neocortical and hippocampal glucose hypometabolism following neurotoxic lesions of the entorhinal and perirhinal cortices in the non-human primate as shown by PET. Implications for Alzheimer's disease. Brain J Neurol. 1999;122 ( Pt 8):1519-1531.

9. Reich MM, Brumberg J, Pozzi NG, et al. Progressive gait ataxia following deep brain stimulation for essential tremor: adverse effect or lack of efficacy? Brain. September 2016:aww223. doi:10.1093/brain/aww223

10. Wintermark M, Huss DS, Shah BB, et al. Thalamic connectivity in patients with essential tremor treated with MR imaging-guided focused ultrasound: in vivo fiber tracking by using diffusiontensor MR imaging. Radiology. 2014;272(1):202-209. doi:10.1148/radiol.14132112

11. Clark LN, Louis ED. Essential tremor. Handb Clin Neurol. 2018;147:229-239. doi:10.1016/B9780-444-63233-3.00015-4

12. Tuleasca C, Witjas T, Najdenovska E, et al. Assessing the clinical outcome of Vim radiosurgery with voxel-based morphometry: visual areas are linked with tremor arrest! Acta Neurochir (Wien). 2017;159(11):2139-2144. doi:10.1007/s00701-017-3317-7

13. Tuleasca C, Witjas T, Van de Ville D, et al. Right Brodmann area 18 predicts tremor arrest after Vim radiosurgery: a voxel-based morphometry study. Acta Neurochir (Wien). November 2017. doi:10.1007/s00701-017-3391-x

14. Tuleasca C, Najdenovska E, Régis J, et al. Pretherapeutic functional neuroimaging predicts tremor arrest after thalamotomy. Acta Neurol Scand. January 2018. doi:10.1111/ane.12891 
15. Tuleasca C, Najdenovska E, Régis J, et al. Clinical response to Vim's thalamic stereotactic radiosurgery for essential tremor is associated with distinctive functional connectivity patterns. Acta Neurochir (Wien). January 2018. doi:10.1007/s00701-017-3456-x

16. Blin J, Mazetti P, Mazoyer B, et al. Does the enhancement of cholinergic neurotransmission influence brain glucose kinetics and clinical symptomatology in progressive supranuclear palsy? Brain J Neurol. 1995;118 ( Pt 6):1485-1495.

17. Ances BM, Vitaliani R, Taylor RA, et al. Treatment-responsive limbic encephalitis identified by neuropil antibodies: MRI and PET correlates. Brain J Neurol. 2005;128(Pt 8):1764-1777. doi:10.1093/brain/awh526

18. Magistretti PJ, Pellerin L. Cellular mechanisms of brain energy metabolism and their relevance to functional brain imaging. Philos Trans R Soc Lond B Biol Sci. 1999;354(1387):1155-1163. doi:10.1098/rstb.1999.0471

19. Gesquière-Dando A, Guedj E, Loundou A, et al. A preoperative metabolic marker of parkinsonian apathy following subthalamic nucleus stimulation. Mov Disord Off J Mov Disord Soc. 2015;30(13):1767-1776. doi:10.1002/mds.26349

20. Lee DS, Kang H, Kim H, et al. Metabolic connectivity by interregional correlation analysis using statistical parametric mapping (SPM) and FDG brain PET; methodological development and patterns of metabolic connectivity in adults. Eur J Nucl Med Mol Imaging. 2008;35(9):16811691. doi:10.1007/s00259-008-0808-z

21. Didic M, Felician O, Gour N, et al. Rhinal hypometabolism on FDG PET in healthy APO-E4 carriers: impact on memory function and metabolic networks. Eur J Nucl Med Mol Imaging. 2015;42(10):1512-1521. doi:10.1007/s00259-015-3057-y

22. Yakushev I, Drzezga A, Habeck C. Metabolic connectivity: methods and applications. Curr Opin Neurol. September 2017. doi:10.1097/WCO.0000000000000494

23. Elble RJ. Diagnostic criteria for essential tremor and differential diagnosis. Neurology. 2000;54(11 Suppl 4):S2-6.

24. Fahn S, Tolosa E, Marin C. Clinical rating scale for tremor.1988:225-234.

25. Llebaria G, Pagonabarraga J, Kulisevsky J, et al. Cut-off score of the Mattis Dementia Rating Scale for screening dementia in Parkinson's disease. Mov Disord. 2008;23(11):1546-1550. doi:10.1002/mds.22173

26. Lieberman MD, Cunningham WA. Type I and Type II error concerns in fMRI research: rebalancing the scale. Soc Cogn Affect Neurosci. 2009;4(4):423-428. doi:10.1093/scan/nsp052

27. Fisher RA. On the probable error of a coefficient of correlation deduced from a small sample. Metron. 1921;1:3-32.

28. Steiger $\mathrm{S}$ James $\mathrm{H}$. Tests for comparing elements of a correlation matrix. Psychol Bull. $1980 ; 87(2): 245$.

29. Stacy MA, Elble RJ, Ondo WG, Wu S-C, Hulihan J, TRS study group. Assessment of interrater and intrarater reliability of the Fahn-Tolosa-Marin Tremor Rating Scale in essential tremor. Mov Disord Off J Mov Disord Soc. 2007;22(6):833-838. doi:10.1002/mds.21412 
30. Régis J, Carron R, Park M. Is radiosurgery a neuromodulation therapy? : A 2009 Fabrikant award lecture. J Neurooncol. 2010;98(2):155-162. doi:10.1007/s11060-010-0226-5

31. Kooshkabadi A, Lunsford LD, Tonetti D, Flickinger JC, Kondziolka D. Gamma Knife thalamotomy for tremor in the magnetic resonance imaging era. J Neurosurg. 2013;118(4):713-718. doi:10.3171/2013.1.JNS121111

32. Benito-León J, Louis ED, Romero JP, et al. Altered Functional Connectivity in Essential Tremor: A Resting-State fMRI Study. Medicine (Baltimore). 2015;94(49):e1936. doi:10.1097/MD.0000000000001936

33. Song I-U, Ha S-W, Yang Y-S, Chung Y-A. Differences in Regional Glucose Metabolism of the Brain Measured with F-18-FDG-PET in Patients with Essential Tremor According to Their Response to Beta-Blockers. Korean J Radiol. 2015;16(5):967-972. doi:10.3348/kjr.2015.16.5.967

34. Vogt BA, Vogt L, Laureys S. Cytology and functionally correlated circuits of human posterior cingulate areas. Neurolmage. 2006;29(2):452-466. doi:10.1016/j.neuroimage.2005.07.048

35. Archer DB, Coombes SA, Chu WT, et al. A widespread visually-sensitive functional network relates to symptoms in essential tremor. Brain J Neurol. 2018;141(2):472-485. doi:10.1093/brain/awx338

36. Bhalsing KS, Kumar KJ, Saini J, Yadav R, Gupta AK, Pal PK. White matter correlates of cognitive impairment in essential tremor. AJNR Am J Neuroradiol. 2015;36(3):448-453.

doi:10.3174/ajnr.A4138

37. Daniels $\mathrm{C}$, Peller M, Wolff S, et al. Voxel-based morphometry shows no decreases in cerebellar gray matter volume in essential tremor. Neurology. 2006;67(8):1452-1456.

doi:10.1212/01.wnl.0000240130.94408.99

38. Spinelli EG, Caso F, Agosta F, et al. A multimodal neuroimaging study of a case of crossed nonfluent/agrammatic primary progressive aphasia. J Neurol. 2015;262(10):2336-2345. doi:10.1007/s00415-015-7845-x

39. Jonas J, Frismand S, Vignal J-P, et al. Right hemispheric dominance of visual phenomena evoked by intracerebral stimulation of the human visual cortex. Hum Brain Mapp. 2014;35(7):33603371. doi:10.1002/hbm.22407 


\section{Figures Legend}

Figure 1. Anatomical localization of areas of decreased metabolism in the entire group of patients with ET after GKVIM in comparison to the baseline evaluation performed before treatment

Anatomical localization of areas of decreased metabolism in the entire group of patients with ET after GKVIM ( $<<0.05$, FWE, $\mathrm{k}>25)$ in comparison to the basal metabolism before GKVIM, projected onto sections of a normal MRI scan centred on the left thalamus (A) and a spatially normalized and smoothed 3D volume rendering (B) on the standard SPM8 template. After GKVIM, patients with ET showed a decreased metabolism in the left thalamus, the left superior and middle temporal gyri (BA 21-22-38), the right cerebellum posterior lobe, the left middle and inferior frontal gyri (BA 10-46) and the right middle and inferior frontal gyri (BA 46). ET: Essential Tremor; GKVIM: Gamma Knife of the Ventral Intermediate Nucleus; MRI: Magnetic Resonance Imaging; BA: Brodmann Area.

Figure 2. Anatomical localization of areas of decreased metabolism in non-responder patients in comparison to responder patients before (A) and after (B) GKVIM and in non-responder patients after GKVIM in comparison to responder patients and the baseline evaluations performed before treatment $(\mathrm{C})$.

Anatomical localization of areas of decreased metabolism in non-responder patients $(p<0.005$, uncorrected, k>150) before (A) and after (B) GKVIM in comparison to responder patients and in non-responder patients after GKVIM ( $\mathrm{p}<0.005$, uncorrected, $\mathrm{k}>150)$ in comparison to responder patients and the baseline evaluations performed before treatment $(\mathrm{C})$, projected onto sections of a normal, spatially normalized and smoothed MRI scan on the standard SPM8 template. In comparison to responder patients, non-responder patients showed decreased metabolism in the right retrosplenium and posterior cingulate (BA 29-30-31) before GKVIM (A) that extended to the right lingual gyrus (BA 18-19) after GKVIM (B). Nonresponder patients also showed decreased metabolism in the right lingual and inferior occipital and fusiform gyri (BA 17-18-19-37) after GKVIM in comparison to responder patients and the basal metabolism found before GKVIM (C). GKVIM: Gamma Knife of the Ventral intermediate nucleus; MRI: Magnetic Resonance Imaging; BA: Brodmann Area. 
Figure 3. Receiver operating characteristic curve for clinical diagnosis of responses with the CMRGIc values of the right retrosplenium and posterior cingulate areas evaluated before treatment

Receiver operating characteristic curve for the clinical diagnosis of responses with the CMRGlc values of the right retrosplenium and posterior cingulate areas (BA 29-30-31) found before treatment. For instance, a mean CMRGlc value of 65 led to $89 \%$ sensitivity and $71 \%$ specificity in predicting response. CMRGlc: Cerebral Metabolic Rate of Glucose; BA: Brodmann Area.

\section{Figure 4. Summary of ${ }^{18}$ F-FDG PET findings}

Preoperative PET showed a metabolic feature involving temporo-occipital hypometabolism and increased connectivity between the thalamus and this temporo-occipital area. This pretherapeutic metabolic feature was predictive of non-response after GKVIM. This predictive pretherapeutic performance was increased by the addition of lesion identification on postoperative MRI. The metabolic changes after left GKVIM involved the left thalamic target and, remotely, a cortico-cerebellar network according to the postoperative PET. 
Figure1
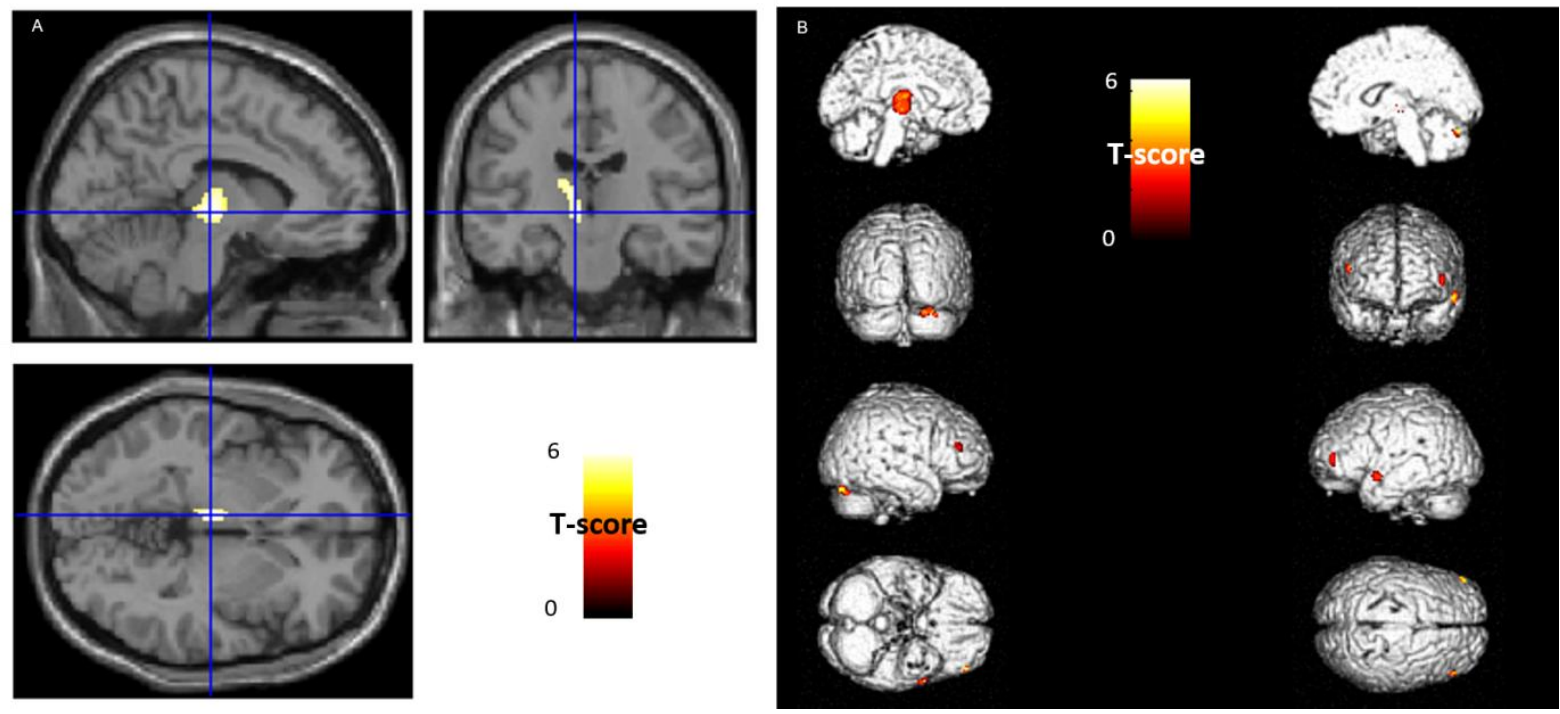

Figure2
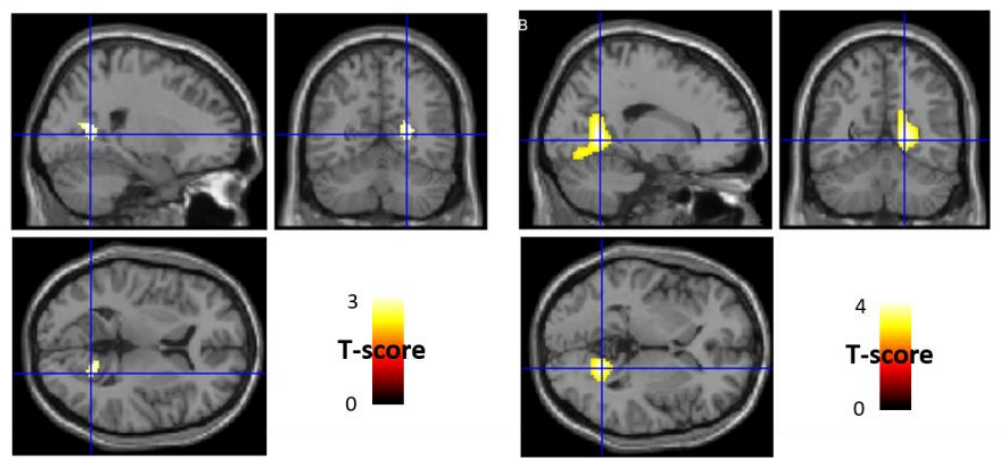

${ }_{0}^{3}$ T-score
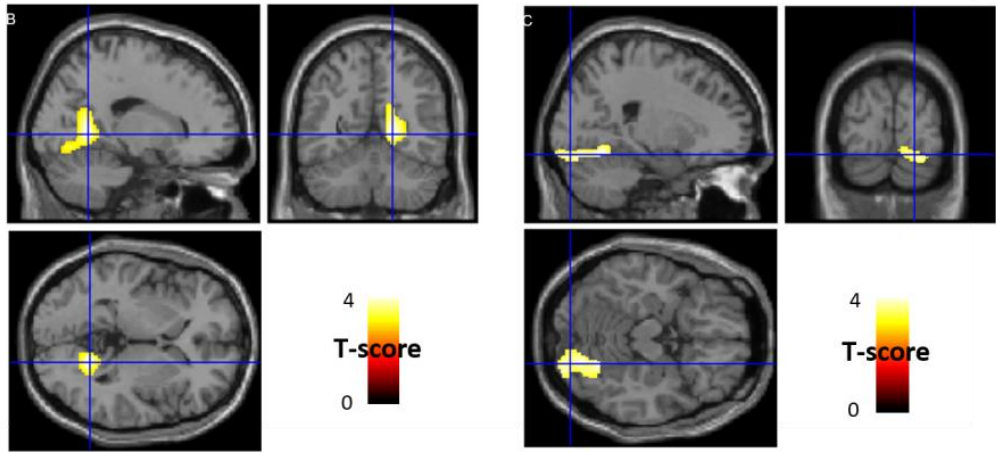


\section{Figure3}

\section{ROC Curve}

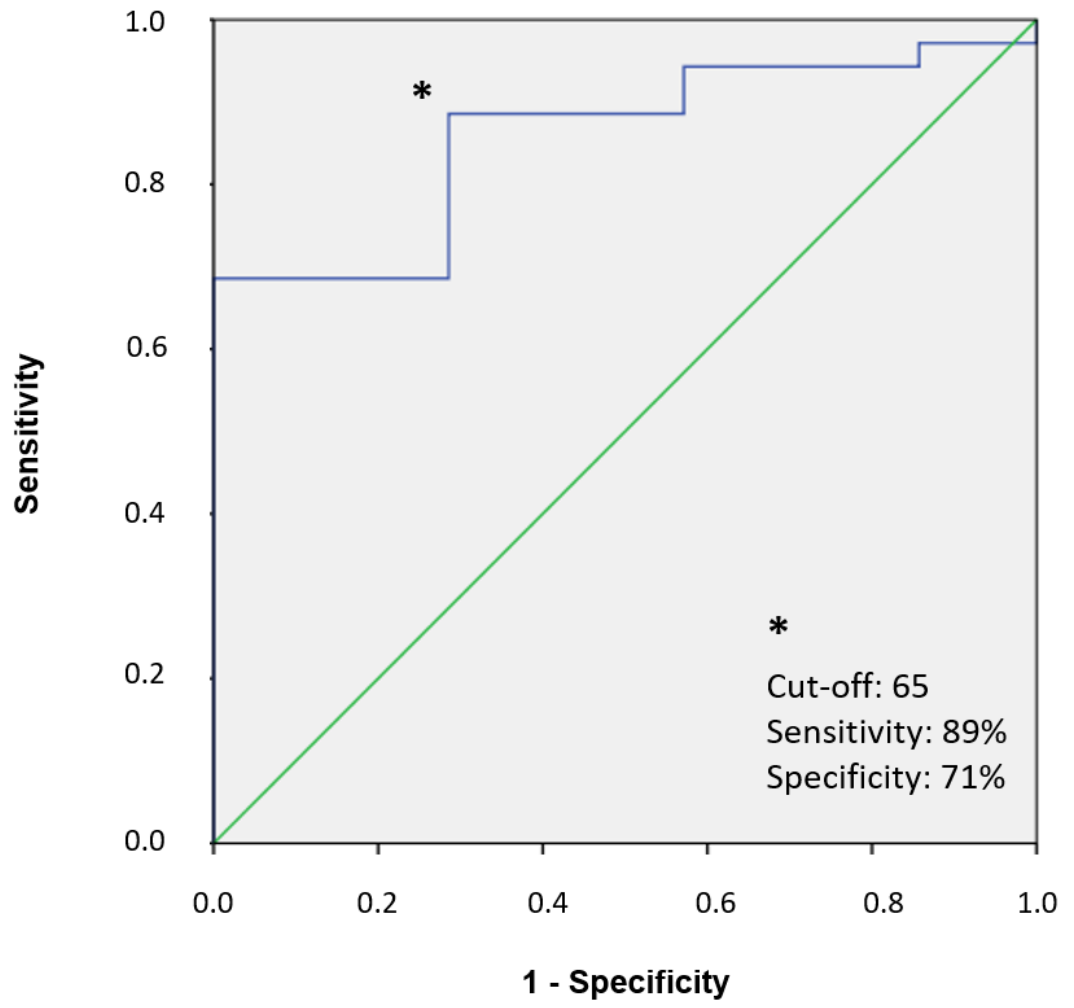

\section{Figure4}

SUMMARY OF ${ }^{18}$ F-FDG PET RESULTS IN PATIENTS WITH ESSENTIAL TREMOR TREATED WITH GKVIM

Preoperative PET

Temporo-occipital

hypometabolism

High connectivity between temporooccipital and thalamic

$$
\text { areas }
$$

Predictive value of non-response to GKVIM

\section{Postoperative PET}

Thalamic

hypometabolism

+ hypometabolism of

bilateral frontal gyri, left

temporal gyri, and right cerebellum

Local and remote effects of GKVIM 
Table 1. Patient characteristics

\begin{tabular}{lccc} 
& $\begin{array}{c}\text { Whole-group } \\
\mathrm{N}=42\end{array}$ & $\begin{array}{c}\text { Responders } \\
\mathrm{N}=35\end{array}$ & $\begin{array}{c}\text { Non-responders } \\
\mathrm{N}=7\end{array}$ \\
\hline Age, years & $71.6(\mathrm{SD}=5.9)$ & $71.3(\mathrm{SD}=5.9)$ & $73.0(\mathrm{SD}=5.9)$ \\
Gender: female & $14(33 \%)^{*}$ & $13(37 \%)^{*}$ & $1(14 \%)^{*}$ \\
Diabetes mellitus (type 2) & $3(7 \%)^{*}$ & $2(6 \%)^{*}$ & $1(14 \%)^{*}$ \\
MDRS total score (/144) before GKVIM & $133.9(\mathrm{SD}=7.6)^{*}$ & $133.3(\mathrm{SD}=7.8)^{\ddagger}$ & $136.5(\mathrm{SD}=6.7)^{\ddagger}$ \\
MDRS total score (/144) after GKVIM & $135.5(\mathrm{SD}=6.6)$ & $135.5(\mathrm{SD}=6.9)$ & $135.3(\mathrm{SD}=5.4)$ \\
Clinical Improvement, \% of treated side & $64.2 \%(\mathrm{SD}=31.8)$ & $76.3 \%(\mathrm{SD}=17.3)$ & - \\
Radiological Responders & $32(76 \%)^{*}$ & $30(86 \%)^{*}$ & $2(29 \%)^{*}$ \\
$\begin{array}{l}\text { Average Delay between pre-surgical } \\
\text { FDG PET and GKVIM, days }\end{array}$ & $60(\mathrm{SD}=84)$ & $54(\mathrm{SD}=82)$ & $87(\mathrm{SD}=96)$ \\
$\begin{array}{l}\text { Average Delay between GKVIM and } \\
\text { post-surgical FDG PET, days }\end{array}$ & $484(\mathrm{SD}=85)$ & $486(\mathrm{SD}=86)$ & $477(88)$ \\
\hline
\end{tabular}

Clinical response defined as $\geq 45 \%$ of improvement according to the Fahn-Tolosa-Marin Tremor Rating Scale. *: Percentage of categorical variables. *: results for 33 patients only (27 responders and 6 non-responders)

MDRS: Mattis Dementia Rating Scale; GKVIM: Gamma-Knife of the Ventral intermedius 
Table 2. ${ }^{18}$ F-FDG-PET findings in whole-group of patients and in non-responders in comparison with responders (Anatomical locations, Spatial extents of significant Clusters, MNI coordinates, maximal z-Scores and Height threshold of peak-voxel)

Peak

Coordinates

\begin{tabular}{|c|c|c|c|c|c|c|}
\hline & $\begin{array}{c}\text { Cluster } \\
\text { Dimension }\end{array}$ & $\mathbf{X}$ & $\mathbf{y}$ & $\bar{z}$ & $\begin{array}{l}z \text {-score } \\
\text { of Peak }\end{array}$ & $\begin{array}{l}\text { P-Value } \\
\text { (FWEcor) }\end{array}$ \\
\hline \multicolumn{7}{|l|}{$\begin{array}{l}\text { Decrease of metabolism after left GKVIM, in } \\
\text { comparison to metabolism before treatment in the } \\
\text { whole group of patients }\end{array}$} \\
\hline Left Thalamus & 256 & -8 & -18 & -2 & 5.94 & $<0.001$ \\
\hline Left superior and middle Temporal gyri (BA 21-22-38) & 58 & -56 & 4 & -10 & 5.49 & $<0.001$ \\
\hline Right Cerebellum posterior lobe & 91 & 24 & -78 & -26 & 5.31 & $<0.001$ \\
\hline Left middle and inferior Frontal gyri (BA 10-46) & 40 & -44 & 48 & 6 & 5.05 & $<0.001$ \\
\hline \multirow[t]{2}{*}{ Right middle and inferior Frontal gyri (BA 46) } & 26 & 52 & 38 & 20 & 5.03 & $<0.001$ \\
\hline & & & & & & $\begin{array}{l}\text { P-Value } \\
\text { (unc) }\end{array}$ \\
\hline \multicolumn{7}{|l|}{$\begin{array}{l}\text { Decreased metabolism in NR patients after } \\
\text { GKVIM, in comparison to } R \text { patients and to basal } \\
\text { metabolism before treatment }\end{array}$} \\
\hline $\begin{array}{l}\text { Right lingual and inferior occipital and fusiform gyri } \\
\text { (BA 17-18-19-37) }\end{array}$ & 809 & 24 & -62 & -18 & 3.71 & $<0.001$ \\
\hline \multicolumn{7}{|l|}{$\begin{array}{l}\text { Hypometabolism in NR patients in comparison to } R \\
\text { patients before treatment }\end{array}$} \\
\hline $\begin{array}{l}\text { Right retrosplenium and posterior cingulate } \\
\text { (BA 29-30-31) }\end{array}$ & 213 & 24 & -60 & 12 & 3.39 & $<0.001$ \\
\hline \multicolumn{7}{|l|}{$\begin{array}{l}\text { Hypometabolism in NR patients in comparison to } R \\
\text { patients after GKVIM }\end{array}$} \\
\hline $\begin{array}{l}\text { Right lingual gyrus, retrosplenium and posterior } \\
\text { cingulate } \\
\text { (BA 18-19-29-30-31) }\end{array}$ & 842 & 18 & -56 & 2 & 4.30 & $<0.001$ \\
\hline
\end{tabular}

Abbreviations: BA: Brodmann Area; FWE: Family Wise Error correction; GKVIM: GammaKnife of the Ventral intermedius nucleus; MNI: Montreal Neurological Institute; NR: NonResponders; R: Responders; unc.: uncorrected 